\title{
Forests and Climate change Le changement climatique et les forêts
}

\author{
Position Paper/Expose de position
}

\section{Canadian Institute of Forestry/Institut forestier du Canada}

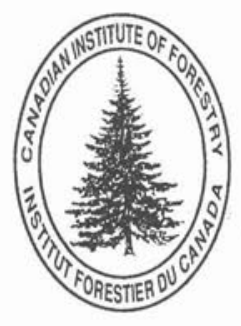

Although climate change has been the object of a great deal of press coverage, it is not a new phenomenon. Indeed, while the world's climate has always warmed and cooled during different epochs due to natural causes, the average temperature of the earth's surface has remained relatively constant over the past 100,000 years. More recently, however, human activities associated with production and consumption activities have increased to a point where they significantly contribute to these natural processes. Humankind's actions have thus become a significant factor in influencing present and future climatic conditions.

The most significant human impact on climate results from the increasing emissions of gases (particularly carbon dioxide, nitrous oxide, methane, chlorofluocarbons and ozone) into the atmosphere. Other impacts include changing land use (e.g. conversion of tropical and temperate forests to Savannah, and agricultural grass lands to deserts) urban heat, jet condensation trails, smoke and dust. Together these impacts have resulted in a global warming of about $0.6^{\circ} \mathrm{C}$ over the past century, and it is predicted that a further rise of 1 to $4.5^{\circ} \mathrm{C}$ will take place by the $2030 \mathrm{~s}$ unless human impacts are greatly reduced immediately. High latitudes in both hemispheres will experience the greatest warming, and the amount of warming will be greatest in the winter months. Thus Canada is expected to bear the brunt of the impact of climate change in North America.

\section{Potential impact of climate change on trees and forest}

1. Canada's tree line may gradually migrate $100 \mathrm{~km}$ northward for every Celsius degree of warming. This should result in a northward movement of the zone of maximum growth of a given tree species.

2. Tree growth may be enhanced in areas where low temperature is now a limiting factor.

3. It is likely that new species, varieties and forms may evolve as a result of climatic change, species migration and exposure to new habitats.

4. Some currently forested areas in Canada, particularly the southern limits of some forest types, may no longer be suitable for the growth of particular indigenous tree species. Maintaining forest cover in these areas may require the introduction of tree species and varieties currently restricted to the United States.

5. The anticipated increase in temperature, and an associated decrease in summer precipitation or modification of the precipitation regime in parts of Canada is likely to increase wildfire activity and intensity.

6. The warming may affect the activity, abundance and distribution of many insects and diseases. A warmer climate will likely expand the distribution of some forest pests to higher latitudes and altitudes.
Bien que le changement climatique ait fait l'objet d'une grande couverture médiatique, il ne s'agit pas d'un phénomène récent. En effet, au cours des époques, le climat de la planète a été marqué par des périodes de réchauffement et de redroidissement. Néanmoins, la température de la surface de la terre est demeurée relativement constante depuis 100000 ans. Ces derniers temps toutefois, les activités humaines liées à la production et à la consommation ont influé sur les processus naturels que sont le réchauffement et le refroidissement. Elles sont devenues, en fait, un facteur déterminant des conditions climatiques actuelles et futures.

Les répercussions les plus importantes de l'activité humaine sur le climat découlent des émissions croissantes de gaz dans l'atmosphère - dioxyde de carbone, oxyde nitreux, méthane, hydrocarbures chlorofluorés et ozone. Parmi les autres facteurs qui influent sur le changement climatique, signalons l'évolution de l'utilisation des sols (p. ex., la transformation des forêts des zones tropicale et tempérée en savannes, et des prairies en déserts), les puits thermiques urbains, les traînées de condensation dans le sillage des avions à réaction, la fumée et la poussière. Collectivement, ces facteurs ont entraîné une hausse de la température de la surface de la terre d'environ $0,6^{\circ} \mathrm{C}$ au cours du siècle dernier. Qui plus est, si l'on ne parvient pas à atténuer sensiblement les répercussions des activités humaines, on peut s'attendre à une hausse additionnelle de 1 à $4,5^{\circ} \mathrm{C}$ d'ici l'an 2030. Le cas " chéant, c'est dans les latitudes polaires que le réchauffement sera le plus prononcé, surtout durant les mois d'hiver. Ainsi, en Amérique du Nord, c'est le Canada qui portera le poids des effets du changement climatique.

\section{Répercussions possibles du changement climatique sur les arbres et les forêts}

1. Chaque augmentation d'un degré Celsius de la température pourrait s'accompagner d'un déplacement de la limite forestière du Canada de quelque $100 \mathrm{~km}$ vers le Nord et du prolongement, vers le Nord également, de l'aire de croissance maximale des essences forestières.

2. La croissance des arbres pourraient être favorisée dans des régions ou les basses rempératures sont présentement un facteur contraignant.

3. Il y a de fortes chances que le changement climatique, la migration d'espèces et l'exposition à de nouveaux habitats entraîneront l'apparition de nouvelles espèces, variétés et formes de végétation.

4. Certaines régions forestières du Canada, notamment les aires les plus australes de certains peuplements forestiers ne sont peut-être plus propices à la croissance d'essences indigènes. La pérennité de la couverture forestière dans ces régions, exigera peut-être l'introduction d'essences et de variétés dont l'aire de croissance est actuellement limitée aux États-Unis.

5. La hausse de la température prévue, la diminution de la précipitation qu'elle entraînera, voire le changement des régimes des précipitations dans certaines parties du Canada 
7. Reduced snow cover and warmer winters may require adjustments in forest harvesting operations, especially when heavy machinery and equipment are used.

8. Changes in the growth and composition of forests and associated ground vegetation will likely influence wildlife population.

9. In the long term Canada's network of ecological reserves and parks may have to be re-evaluated because of changing ecosystems.

The CIF/IFC recommends that:

1. the scientific community utilize appropriate climatic change models to provide estimates of the potential impact of global warming on forest dynamics such as growth and biodiversity;

2. the scientific community assesses the adaptability of Canada's forest ecosystems to climate change, particularly with regard to sustainability; and,

3. the forest sector takes measures to mitigate the effect of climate change, particularly by addressing the carbon budget of the Canadian Forest Sector through application appropriate silvicultural and management activities;

4. the scientific community work with the forest sector to develop appropriate sustainable forest management strategies to help ameliorate the predicted problems arising from climate change.

\section{Who we are}

The Canadian Institute of Forestry is a national, non-profit forestry organization. Our mission is to advance the stewardship of Canada's forest resources, provide national leadership in forestry, promote competence among forestry professionals, and foster public awareness of Canadian and international forestry issues.

We are people with a professional interest in forestry, working in government, industry, academic and consulting fields. Our members use their education, training, and experience to help manage the forests of Canada and to make the Canadian public aware of forestry.

This is a statement of what we stand for - the Institute's position on Forests and Climate Change.

Canadian Institute of Forestry/Institut forestier du Canada, 151 Slater Street, Suite 606, Ottawa Ontario K1P 5H3. Telephone: 613-234-2242, Facsimile: 613-234-6181, Email: cif@cififc.org, Internet: http://www.cif-ifc.org

\section{Prepared by Dr. L.C. Duchesne, Chair Ecology Working} Group

Amended and Approved by the Executive Committee, October, 1997 pourraient intensifier l'incidence et l'intensité des incendies de forêt.

6. Le réchauffement pourrait influer sur l'activité, l'abondance et la distribution d'un grand nombre d'insectes et maladies. Le réchauffement du climat contribuera tout probablement à l'expansion de l'aire de distribution des ravageurs forestiers vers les latitudes septentrionales et les altitudes supérieures.

7. La réduction de l'enneigement et les hivers moins rigoureux pourraient entraîner une modification des techniques d'exploitation forestière, notamment dans les régions où l'emploi de machinerie lourde est répandu.

8. Le changement climatique influera sur la croissance et la composition des forêts et de la litière végétale et ces changements influeront à leur tour sur la faune.

9. À plus long échéance, il faudra peut-être repenser le réseau des réserves écologiques et des parcs du Canada en raison de l'évolution des écosystèmes.

En raison des répercussions possibles susmentionnées, l'IFC/CIF formule les recommendations suivantes:

1) QUE la collectivité scientifique utilise des modèles de changement climatique appropriés pour établir des prévisions concernant les répercussions du réchauffement de la planète sur la dynamique forestière, notamment la croissance et la diversité biologique;

2) QUE la collectivité scientifique évalue l'adaptabilité des écosystèmes forestiers du Canada au changement climatique, surtout en ce qui a trait à leur pérennité;

3) QUE le secteur forestier prenne les mesures nécessaires pour atténuer les répercussions du changement climatique, particulièrement en influant sur le bilan du carbone du secteur forestier du Canada au moyen d'activités sylvicoles et d'aménagement forestier appropriées;

4) QUE la collectivité scientifique, en collaboration avec le secteur forestier, mette au point des stratégies d'aménagement forestier durable susceptibles d'atténuer les problèmes qui, selon les prévisions, se manifesteront par suite du changement climatique.

\section{À propos de l'institut}

L'Institut forestier du Canada est un organisme sans but lucratif national ayant pour mission de faire progresser la festion des ressources forestière au Canada grâce à son leadership, à la compétence professionnelle de ses membres et à ses initiatives de sensibilisation du public aux enjeux forestiers, tant à l'échelle nationale qu'à l'échelle internationale.

Oeuvrant dans la fonction publique, le secteur privé, l'enseignement et à titre d'experts-conseils, les membres de l'Institut portent un intérêt professionnel à tout ce qui touche à la foresterie. Ils mettent leur éducation, leur formation et leur expérience à profit pour faire progresser l'aménagement forestier au Canada et pour sensibiliser la population en matière de foresterie.

La présente brochure est un exposé de la position de l'Institut sur le changement climatique et ses incidences sur les forêts.

Institut forestier du Canada/Canadian Institute of Forestry, 151, rue Slater, bureau 606, Ottawa (Ontario) K1P 5H3. Téléphone: (613) 234-2242, Télécopieur: (613) 234-6181, CÉ: cif@cif-ifc.org, Internet: http://www.cif-ifc.org.

Rédaction: Dr. L.C. Duchesne, président, Groupe de travail sur l'écologie forestière

Révisé et approuvé par le Comité de direction Octobre 1997 